\section{Needle-Stick Injury Caused by a Patient With Severe Fever With Thrombocytopenia Syndrome in Korea}

To the Editor-Several studies have identified clusters of severe fever with thrombocytopenic syndrome (SFTS) infections among family members and that appear to have been transmitted by human contact. ${ }^{1-6}$ In addition, possible transmission from the index patient with SFTS to healthcare workers (HCWs) has been reported. ${ }^{1,3,7}$ Blood and body fluids were suggested as the possible transmission route. Therefore, strict adherence to routine blood and body fluid precautions is required when HCWs come into contact with any patient, especially anyone with suspected viral hemorrhagic fever or a tick-borne rickettsial disease. Herein we report the result of needle-stick injury to an HCW caused by a patient with a high viral load of SFTS.

A 62-year-old woman was admitted to Asan Medical Center with a 5-day fever, myalgia, and a headache (July 1, 2015). On her hospital day (HD) 6, her blood pressure decreased to 80/45 $\mathrm{mm} \mathrm{Hg}$ and her condition rapidly declined, causing a need for mechanical ventilation. The patient was admitted to the intensive care unit. On HD 7, SFTS-associated encephalopathy was diagnosed from detection of SFTS virus (SFTSV) by real-time polymerase chain reaction assay (RT-PCR) from plasma and cerebrospinal fluid. On HD 12, a nurse who took care of the patient experienced needle-stick injury on her finger during blood sampling. The needle was filled with the patient's blood. The needle penetrated her left third finger skin with a notable amount of bleeding. The viral load of the patient was $1 \times 10^{5}$ on HD 13. Four days later (July 16, 2015), we checked her blood for SFTSV by RT-PCR and immunofluorescence assay titer for evaluation of possible SFTSV transmission, despite no development of symptoms, because there was a previous report of subclinical infection in $1 \mathrm{HCW}$ who had contacted an index patient. ${ }^{6}$ SFTSV was not found by RT-PCR and the total immunoglobulin G level for SFTSV immunofluorescence assay was less than 1:32. The HCW had not developed any symptoms 6 weeks after the needle-stick injury. Her convalescent serum drawn 1 month after the injury recheck (August 13, 2015) revealed SFTSV was not detected by RT-PCR and the total immunoglobulin G level for SFTSV immunofluorescence assay was less than 1:32.

Recently, viral hemorrhagic fevers, such as Ebola virus disease, have attracted renewed attention owing to the large Ebola virus disease outbreak in West Africa. ${ }^{8}$ Therefore, nosocomial transmission to HCWs from patients with suspected viral hemorrhagic fever is of paramount importance. SFTSV is a third group within the genus phlebovirus, family Bunyaviridae, one of the 5 families causing viral hemorrhagic fever. ${ }^{4}$ Interestingly, the overall transmission rate of SFTS in a previous study by our group ${ }^{7}$ was $15 \%$, which is comparable to the household transmission rate $(16 \%)$ of Ebola virus disease in the absence of personal protective devices. ${ }^{9}$ Therefore, it is theoretically possible that direct inoculation of blood that contains sufficient volumes of infectious SFTS can cause infection via needle-stick injury. Fortunately, SFTSV was not transmitted in our case despite the high viral load in the index patient's blood. However, the possible effects of needle-stick injury, such as Ebola virus disease transmission, deserve further scrutiny.

\section{ACKNOWLEDGMENTS}

Financial support. The National Research Foundation, funded by the Ministry of Education, Science and Technology (grant 2013R1A1A1A05004354); and the Korea Centers for Disease Control and Prevention (grant 4800-4837301).

Potential conflicts of interest. All authors report no conflicts of interest relevant to this article.

Se Yoon Park, MD;
Sung-Han Kim, MD, PhD;
Sun-Whan Park, PhD; ${ }^{2}$
Eun Byeol Wang, BSc;
Won Ja Lee, PhD;
Youngmee Jee, MD, PhD;
WooYoung Choi, PhD $^{2}$

Affiliations: 1. Department of Infectious Disease, Asan Medical Center, University of Ulsan College of Medicine, Seoul, Republic of Korea; 2. Division of Arboviruses, National Institute of Health, Korea Centers for Disease Control and Prevention, Choungcheonbuk-do, Republic of Korea.

Address correspondence to WooYoung Choi, Division of Arboviruses, National Institute of Health, Cheongju, Korea (wychoi65@nih.go.kr). Infect. Control Hosp. Epidemiol. 2016;37(3):368-369

(C) 2016 by The Society for Healthcare Epidemiology of America. All rights reserved. 0899-823X/2016/3703-0027. DOI: 10.1017/ice.2015.322

\section{REFERENCES}

1. Gai Z, Liang M, Zhang Y, et al. Person-to-person transmission of severe fever with thrombocytopenia syndrome bunyavirus through blood contact. Clin Infect Dis 2012;54:249-252.

2. Bao CJ, Guo XL, Qi X, et al. A family cluster of infections by a newly recognized bunyavirus in eastern China, 2007: further evidence of person-to-person transmission. Clin Infect Dis 2011; 53:1208-1214.

3. Liu Y, Li Q, Hu W, et al. Person-to-person transmission of severe fever with thrombocytopenia syndrome virus. Vector Borne Zoonotic Dis 2012;12:156-160.

4. Tang $\mathrm{X}, \mathrm{Wu} \mathrm{W}$, Wang $\mathrm{H}$, et al. Human-to-human transmission of severe fever with thrombocytopenia syndrome bunyavirus through contact with infectious blood. I Infect Dis 2013;207: 736-739.

5. Chen $\mathrm{H}, \mathrm{Hu} \mathrm{K}$, Zou J, Xiao J. A cluster of cases of human-tohuman transmission caused by severe fever with thrombocytopenia syndrome bunyavirus. Int J Infect Dis 2013;17:e206-e208. 
6. Wang Y, Deng B, Zhang J, Cui W, Yao W, Liu P. Personto-person asymptomatic infection of severe fever with thrombocytopenia syndrome virus through blood contact. Intern Med 2014;53:903-906.

7. Kim WY, Choi W, Park SW, et al. Nosocomial transmission of severe fever with thrombocytopenia syndrome in Korea. Clin Infect Dis 2015;60:1681-1683.
8. WHO Ebola Response Team. Ebola virus disease in West Africa: the first 9 months of the epidemic and forward projections. N Engl J Med 2014;371:1481-1495.

9. Dowell SF, Mukunu R, Ksiazek TG, Khan AS, Rollin PE, Peters CJ. Transmission of Ebola hemorrhagic fever: a study of risk factors in family members, Kikwit, Democratic Republic of Congo, 1995. J Infect Dis 1999;179:S87-S91. 